\title{
Digitizing Entire Populations of Consumers for Smartphone Applications
}

\author{
David BRUNER \\ $[\mathrm{TC}]^{2}$, Textile/Clothing Technology Corp., Cary (NC), USA
}

\begin{abstract}
The ability to create digital representations of objects is a product of computer age technologies. Use of these representations has exploded with the evolution of internet applications. Now, with wide availability of "smart phones" the internet is always with you. Perhaps the most important digital object for a consumer is the digital representation of themselves, either as photos, or as three-dimensional "Avatars". These representations are the virtual representative for the individual when they participate in social networks or "Virtual Worlds". Other than being your online representation which others view you, there is no requirement or need that the representation be "true". In fact, much of the time a representative digital photograph or avatar is chosen in a form that is not true to your actual physical configuration. There is however a subset of applications of personal digital data where it is crucial that the data be "true" or correct. Online selection and viewing of apparel or applications related to personal health and fitness are two examples.

There are many forms of human body digital representation. These forms can be as simple as the digital photo or a handful of linear body measurements as numeric values. They can be as complex as color-textured 3D body scan data consisting of many millions of data points. In addition to quantity of digital data is also the question of quality/accuracy of that data. From simple to complex all of these digital forms have some degree of value in application. It is the application which determines the required amount of data and accuracy. Generally speaking, higher cost is associated both with "more data" and "more accurate" data. That cost is not static and is decreasing dramatically over time, inspiring the possibility to digitize entire populations and provide access to that data for consumers. This is enabling a wide range of applications available to consumers and expansion of online commerce related to these applications.
\end{abstract}

\section{Summary}

\section{Digitization Techniques}

1. Photography

2. Manual Measurements
a. Self
b. Professional

3. Silhouette Photos and Measurements

4. 3D Body Scanning with Automatic Measurement and Avatar Creation
a. Full body scanning machines
b. Scanning at home with low cost consumer depth sensors (Kinect and others)
c. Full body scan machines made from low cost consumer depth sensors

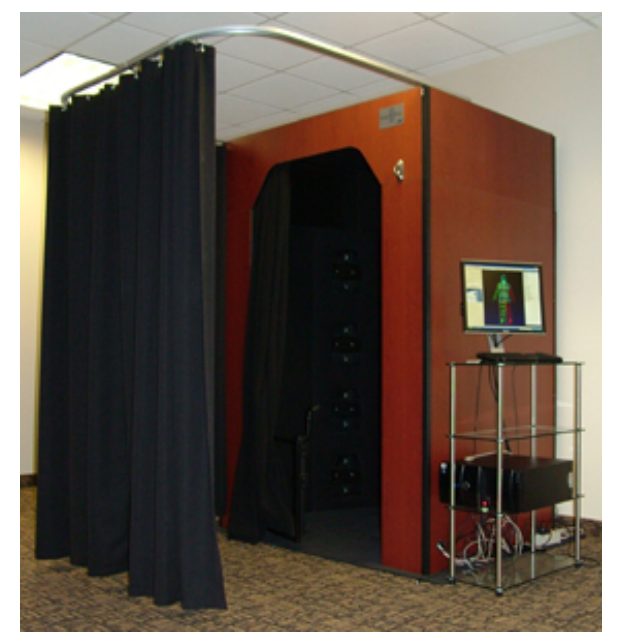

KX-16 Low Cost 3D Body Scan booth using Kinect Sensors 


\section{Human Body Digitization Components}

1. Body Geometry

2. Face detail and skin texture

3. Hairstyle and Color
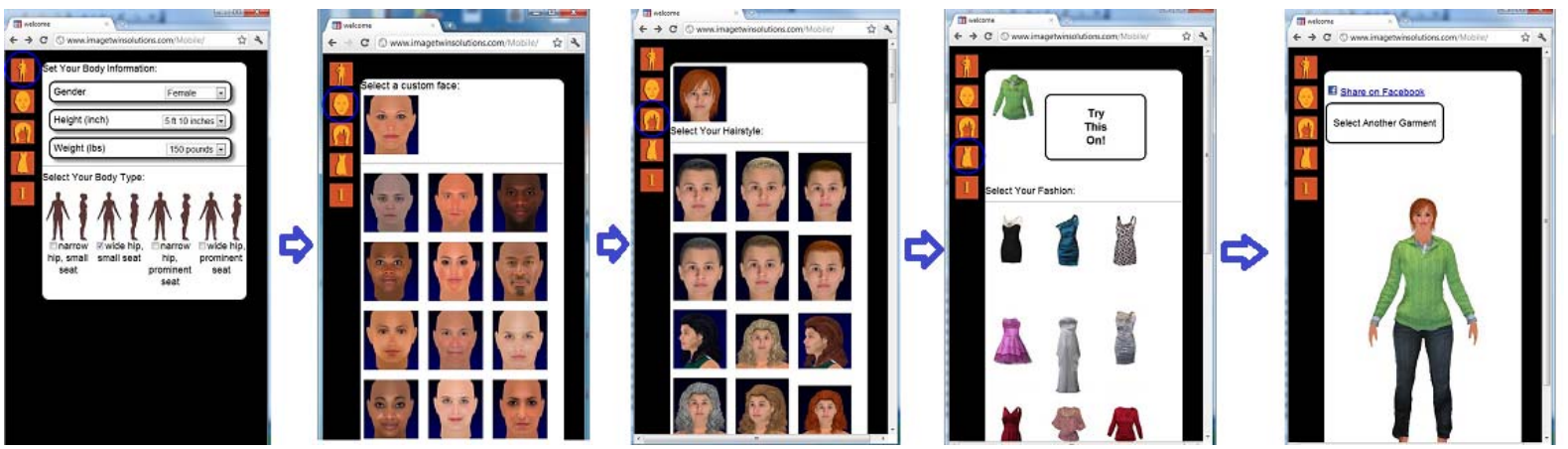

Example Smart Phone Application: Virtual Fashion, Virtual dressing, and size selection from digital body data

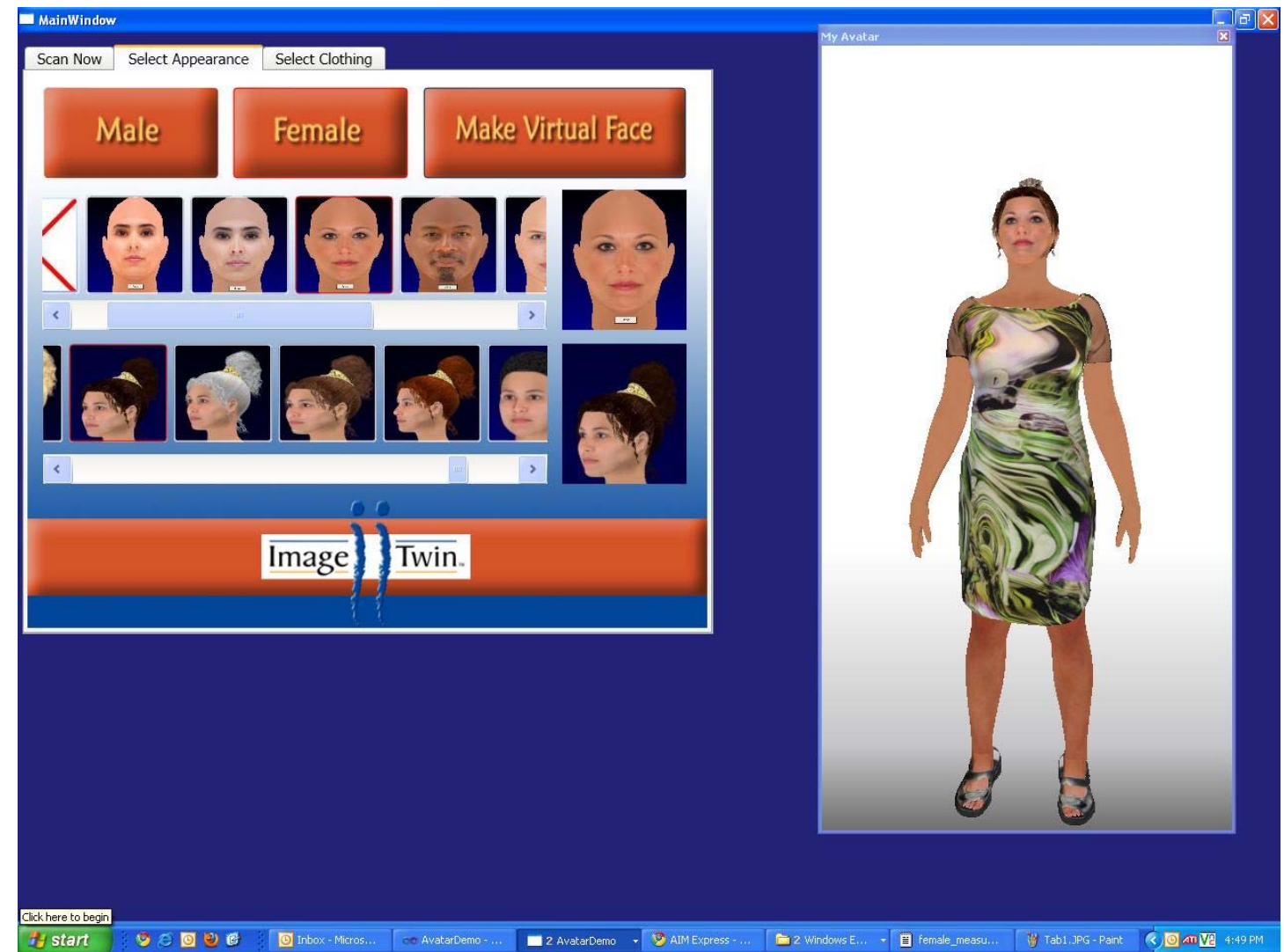

Example Kiosk Application for Virtual Fashion

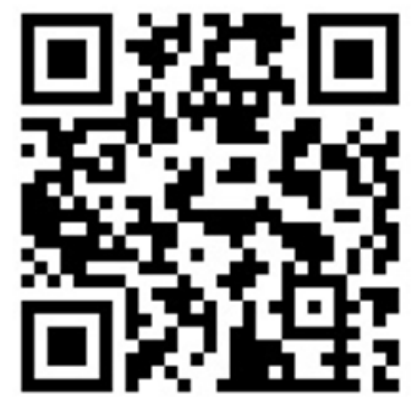

Demonstration Website Link Through QR Code 